\title{
The PEP-II Project: Low-Energy Ring Design and Project Status
}

\author{
Michael S. Zisman* \\ Accelerator \& Fusion Research Division \\ Ernest O. Lawrence Berkeley National Laboratory \\ Berkeley, CA 94720 U.S.A.
}

\begin{abstract}
We describe the present status of the PEP-II project. The project comprises four major systems: Injector, High-Energy Ring (HER), Low-Energy Ring (LER), and Interaction Region (IR). We focus in detail on the design of the LER, as its parameters and requirements are most closely related to those required for the Beijing Tau-Charm Factory rings. The PEP-II LER is a high-current, 3.1-GeV positron ring mounted above the 9-GeV HER. The LER uses a wiggler located in one of its six straight sections to provide emittance control and additional damping. We describe the rather complicated IR, which must transport the LER beam into the plane of the HER, focus it to a common beam size, and separate the beams after the head-on collisions. Both permanent magnet and conventional electromagnets are used in this area. The LER lattice has now adopted a simplified non-interleaved sextupole correction scheme that has reduced the required number of sextupoles substantially. We describe the LER vacuum system, one of the most challenging subsystems in PEP-II. It employs several technologies. In the arcs, aluminum extrusions and titanium sublimation pumps are employed; the straight sections use stainless steel chambers with lumped ion pumps. In the wiggler area, an extended copper photon dump with nonevaporable getter (NEG) pumps is employed to handle the very large synchrotron radiation power. The design of the room-temperature RF system, the bunch-by-bunch longitudinal and transverse feedback systems, and some of the special diagnostics will be described briefly. The PEP-II project remains on schedule to begin commissioning of the HER in April 1997, followed by the LER a year later.
\end{abstract}

\section{INTRODUCTION}

The purpose of the PEP-II Asymmetric $B$ Factory project [1] is to perform experiments to determine the origins of the phenomenon of charge-conjugation-parity $(C P)$ violation. The facility will additionally be able to study a broad range of high-energy physics issues in the energy region near the $\Upsilon(4 \mathrm{~S})$ resonance. To carry out its primary function, PEP-II requires two novel features. Firstly, it must provide very high integrated luminosity. The design goal is to provide a peak luminosity of $3 \infty 10^{33} \mathrm{~cm}^{-2} \mathrm{~s}^{-1}$ and an integrated luminosity (over a "Snowmass year" of $10^{7} \mathrm{~s}$ ) of $30 \mathrm{fb}^{-1}$. Secondly, we make use of asymmetric beam energies, that is, the electron and positron energies are unequal. For PEP-II, we choose an electron energy of $9 \mathrm{GeV}$, and a positron energy of $3.1 \mathrm{GeV}$. Clearly, this feature requires that PEP-II be a two-ring collider. We have specified that the collisions be head-on at the startup of the machine, though allowance for a non-zero crossing angle has been made in the IR layout to allow a future upgrade path. The initial design studies for the PEP-II project began in 1988, though the project was not funded until January 1994. PEP-II is being built by a tri-lab collaboration of SLAC, Berkeley Lab, and LLNL; this collaboration began formally in December 1989.

\footnotetext{
*Work supported by the U.S. Department of Energy under contract number DE-AC03-76SF00098.
} 
The PEP-II project includes four major systems: Injector, HER, LER, and IR. The Injector includes the extraction and transport lines from the SLAC two-mile linac. With its damping rings, the SLAC linac, which presently operates as the Stanford Linear Collider (SLC), is the world's most powerful positron source. The HER is mainly a refurbished PEP ring. We have reused all the PEP magnets and some of the power supplies, but are providing new vacuum, RF, feedback, and diagnostics systems. The LER is an entirely new ring, so nearly all components must be newly fabricated. The IR, which is also new, is the most intricate part of the machine. Not only are there complicated interfaces with the two rings, it must satisfy the additional requirement of compatibility with the $B A B A R$ detector.

As might be expected, there are many technical systems common to both rings, including power supplies, RF systems, feedback systems, and most of the diagnostics. This approach has saved design effort and, in some cases, permits the savings associated with volume purchases. Clearly, however, the very different beam energies of the two rings lead to different technical choices in some instances. In this paper, we focus mainly on the systems used by the LER, as these are most relevant to the future needs of the Beijing TauCharm Factory (BTCF).

The strategy adopted for completion of PEP-II is to undertake a phased commissioning process. First we are fabricating and commissioning the injection lines. This activity is now under way, with the electron extraction from the linac and transport to the end of the linac in a bypass line already accomplished. The positron extraction line is being prepared for completion later this year. Next, we complete the HER in April 1997 and begin to study ring injection and high-current multibunch operation. Finally, the LER is completed in April 1998. We will first study single-ring issues for the LER and then bring the beams into collision and begin studying beam-beam issues. Though the $B A B A R$ detector will not be on line until early 1999, the final IR will be in place for the LER commissioning

\section{LER DESIGN FEATURES}

The PEP-II LER will be one of the highest current positron rings ever built. It will operate with about ten times the current of the original PEP ring. As will be obvious from the discussion below, the LER design has much in common with the requirements of the BTCF rings. Main parameters for both PEP-II rings are summarized in Table 1.

The LER is nominally a 3.1-GeV positron ring. As illustrated in Fig. 1, the beam circulates counterclockwise, opposite to the direction of the HER beam. In most areas of the ring, the LER is mounted $89 \mathrm{~cm}$ above the plane of the HER. This choice facilitates installation, alignment, and maintenance of the ring compared with a side-by-side arrangement in the tunnel. A prototype LER raft arrangement is shown in Fig. 2. Injection of the LER will take place in the vertical plane, in IR-8.

To bring the LER beam into head-on collision with the HER beam requires rather complicated IR gyrations. The LER beam is brought down into the plane of the HER in

TABLE 1. PEP-II main parameters.

\begin{tabular}{lll}
\hline & HER & LER \\
Energy [GeV] & 9.0 & 3.1 \\
Circumference [m] & 2200 & 2200 \\
Emittance, $y / x[\mathrm{~nm}-\mathrm{rad}]$ & $1.5 / 49$ & $2.0 / 66$ \\
Beta function at IP, $y / x[\mathrm{~cm}]$ & $2.0 / 66$ & $1.5 / 50$ \\
Beam-beam tune shift & 0.03 & 0.03 \\
RF frequency [MHz] & 476 & 476 \\
RF voltage [MV] & 14.0 & 5.1 \\
Bunch length, rms [cm] & 1.2 & 1.0 \\
No. of bunches & 1658 & 1658 \\
Total current $[\mathrm{A}]$ & 1.0 & 2.1 \\
Energy loss per turn $[\mathrm{MeV}]$ & 3.6 & 0.8 \\
\hline
\end{tabular}




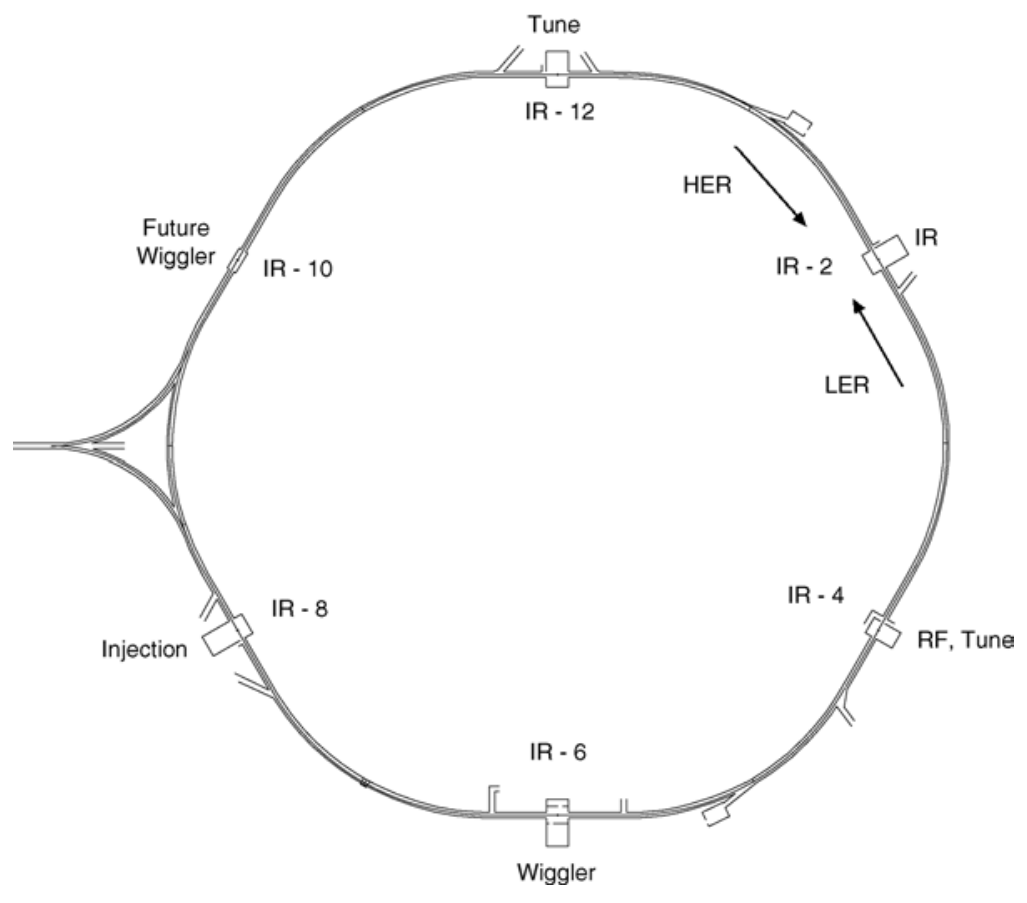

FIGURE 1. Diagram of the PEP-II ring layout. The Interaction Regions are numbered according to the clock. The BABAR detector is located in IR-2.

IR-2. The beams are combined and separated by a pair of dipoles that bend in opposite directions on the two sides of the interaction point (IP), a so-called "S-bend" scheme. This configuration lends itself well to eventual conversion to a non-zero crossing angle geometry. The common quadrupole magnets are also offset from the LER beam axis and provide additional separation.

\section{New Ring Features}

To control the emittance of the LER, and to somewhat increase the radiation damping rate as well, the LER lattice contains a wiggler section in IR-6. We believe that the ability to control the ring emittance in a flexible manner will be important in accommodating the beam-beam behavior in this new collider parameter regime, thus improving our ability to

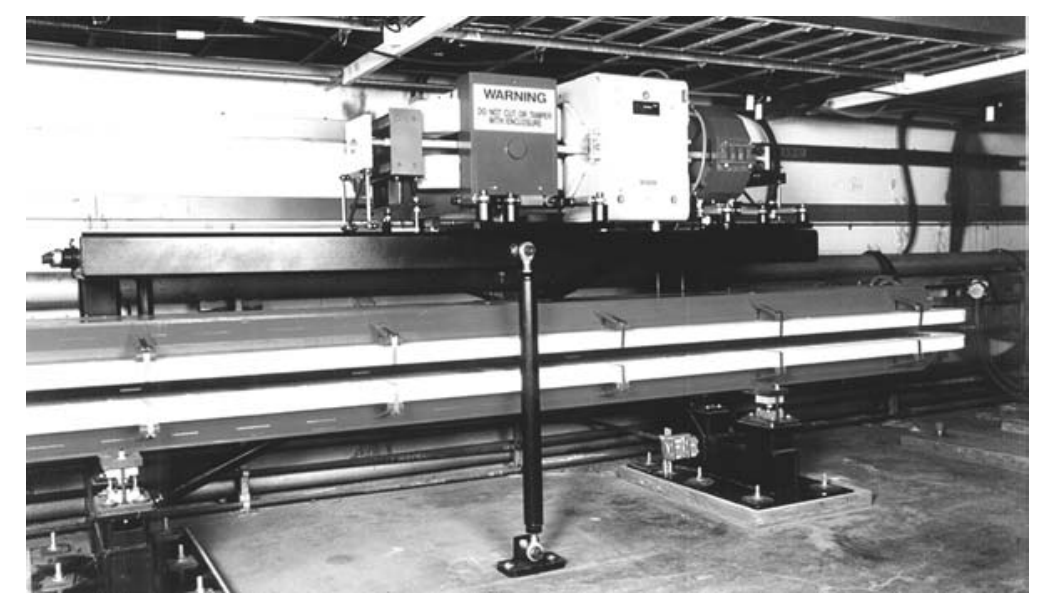

FIGURE 2. Prototype LER arc raft mounted above HER dipole in PEP tunnel. 
reach our design luminosity reasonably quickly. The wiggler is located in a chicane section to facilitate the handling of up to $260 \mathrm{~kW}$ of synchrotron radiation power. A similar chicane section is located in IR-10, to serve as a site for a second wiggler should one be desired in the future. Recently we have evaluated the possibility of reusing the original PEP wiggler units. Although their magnetic length is shorter than our baseline wiggler, they operate at a higher field $(1.8 \mathrm{~T})$ and thus provide full emittance control and nearly full damping rate compared with the baseline design. We have now decided to refurbish the original wiggler units and install them in IR-6.

Another new feature added to both the LER and HER is a beam abort kicker and beam dump system to protect the detector and the vacuum chambers from damage. In each ring, this equipment shares the injection straight section (IR-8 for the LER, IR-10 for the HER). The abort kicker magnet is identical to the injection kicker, but it is driven by a different pulser to give a risetime compatible with the ion clearing gap (about $300 \mathrm{~ns}$ ) and a fall time of one turn $(7.3 \mu \mathrm{s})$. The dump is designed to absorb the full beam power of $66 \mathrm{~kJ}$ (LER) or $200 \mathrm{~kJ}$ (HER).

\section{Interaction Region}

As noted, the IR (see Fig. 3) is the most intricate part of the ring. It has many functional requirements to meet:

- focusing unequal energy beams to a common beam spot

- bringing two beams into head-on collision and then separating them cleanly

- providing adequate beam separation during injection

- providing stable support for the ring components without interfering with detector access or solid-angle coverage

- providing low pressure to minimize beam-gas scattering

- providing masking to limit detector backgrounds

- providing clearance for luminosity monitoring

The LER beam is focused by the quadrupole doublet Q1 and Q2. The Q1 magnet is common to both LER and HER beams. Though Q1 is relatively weak for the HER beam, it nonetheless gives a significant reduction in the vertical beta function at Q4, reducing the overall chromaticity of the HER and improving its dynamic aperture.

To combine and separate the beams, the IR utilizes a pair of dipole magnets, B1, followed by offset quadrupole magnets, Q1. The B1 magnets are tapered to give maximum strength with minimum loss of detector solid-angle coverage. Because all of these magnets are immersed in the solenoidal field of the detector, we use permanent magnet technology. There are two separation criteria that must be met. First, we demand adequate separation $\left(>7 \sigma_{x}\right)$ at the first parasitic crossing points, which occur $63 \mathrm{~cm}$ from the IP. Second, we demand adequate separation between the two beam stay clear regions [2] to accommodate the septum of the Q2 quadrupole, which acts only on the LER beam. We have chosen not to make the B1 magnets adjustable, but we have added trim windings on the Q1 magnet (an air-core quadrupole winding) to permit a 3\% strength adjustment on Q1.

Because alignment of the magnetic elements and masks in the IR is crucial to good luminosity performance, the elements within the detector are prealigned in a support tube. The support tube itself can be adjusted with movers to ensure optimum luminosity performance with minimal backgrounds. 


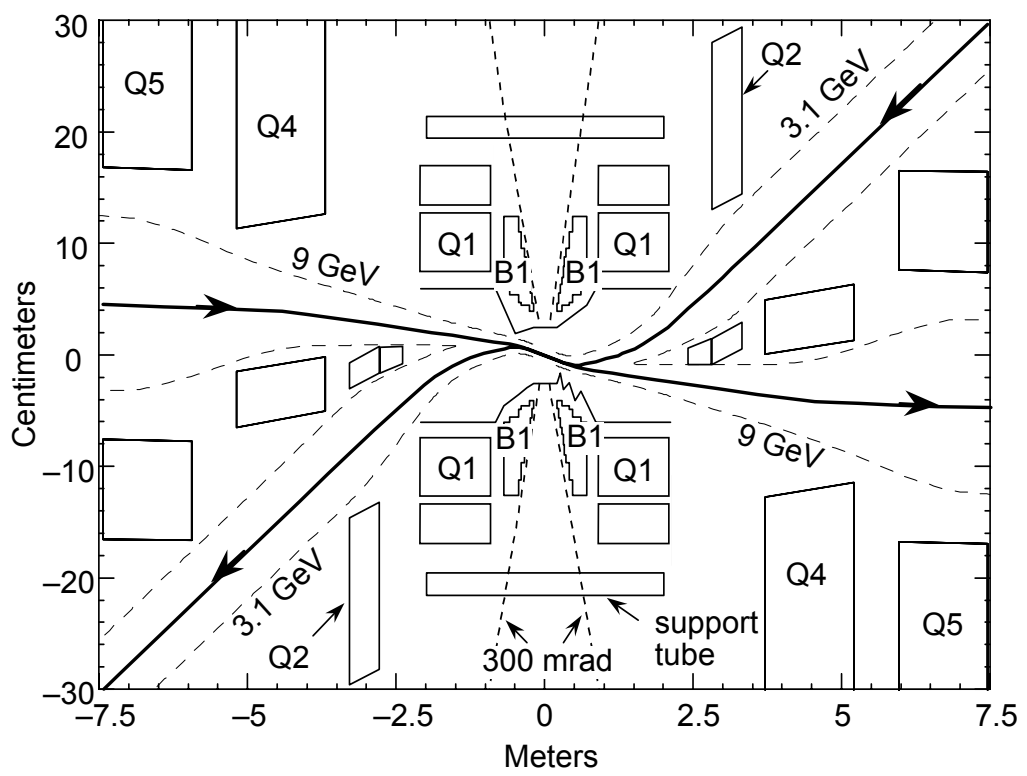

FIGURE 3. Anamorphic diagram of PEP-II interaction region. The beams collide head-on at the IP.

It is worth noting here that one significant design issue for the IR is the close proximity of the two beamlines. Our studies show that magnetic shielding between the elements of the two rings is necessary to avoid degradation of the dynamic aperture. That is, both the Q2 fringe field on the HER beam and the Q4 fringe field on the LER beam must be dealt with.

\section{Lattice}

The LER arcs have a FODO structure with $90^{\circ}$ phase advance in both planes. For ease in installation, the dipoles are not equally spaced between quadrupoles. Rather, the magnets in a half-cell (dipole, quadrupole, sextupole, and corrector) are located together on a common "raft." To enhance the radiation damping, we use relatively short $(45 \mathrm{~cm})$ dipoles. Chromatic correction for the ring is accomplished with a "semi-local" scheme. That is, we use sextupoles within the IR itself and families in the adjacent arc sections to deal with the large chromaticity from the IR along with additional, non-interleaved, sextupole families in the four far arcs to cancel the remaining chromaticity from the arcs and other straight sections. The resulting dynamic aperture is acceptable.

\section{Vacuum System}

Several technologies are employed to construct the vacuum system for the LER. The arcs (see Fig. 4) consist of two types of aluminum extrusions, a magnet chamber and a pumping chamber. The magnet chamber spans the raft holding all the half-cell magnets. It has a slot $15 \mathrm{~mm}$ high to permit the synchrotron radiation fan from the dipole to escape. The much larger pumping chamber has a similar slot that leads to an antechamber containing a Glidcop photon stop and a titanium sublimation pump (TSP). Each photon stop is designed to handle up to $15 \mathrm{~kW}$ of synchrotron radiation power. The close proximity of the highspeed TSP to the localized gas load from the photon stop leads to very low pressure in the 

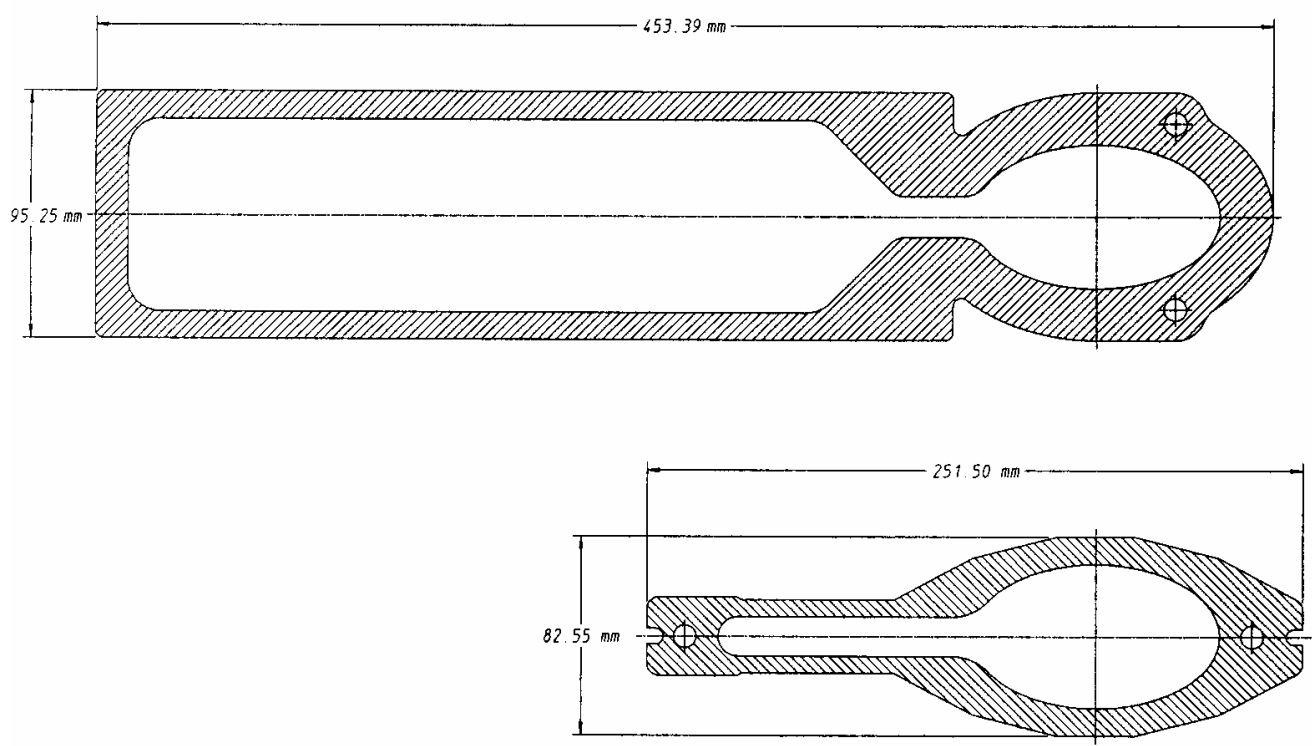

FIGURE 4. Pumping chamber extrusion (top) ; magnet chamber extrusion (bottom).

arcs, about 2 nTorr $\left(\mathrm{N}_{2}\right.$ equivalent). This low pressure is beneficial for maintaining a long beam lifetime in the LER; similar benefits would accrue to a Tau-Charm Factory. Because of the high secondary electron emission coefficient of an aluminum chamber, we plan to coat the inside of both arc chambers with TiN, a material with good mechanical properties and a low secondary emission coefficient. This choice is expected to reduce our sensitivity to the so-called "electron cloud" instability [3].

The standard straight sections use stainless steel tubing. The beam tubes are water cooled to handle the $I^{2} R$ and higher-order-mode (HOM) power. The quadrupole chambers are copper coated on the outside to permit efficient heat transfer to the cooling tubes. At each quadrupole, a screened pump tee and lumped ion pump are provided. Connections between the arc and straight sections are made via elliptical-to-round aluminum transition pieces with appropriate RF smoothness.

In the wiggler chicane, IR-6, we utilize an extended copper photon dump [4]. This chamber must maintain a good vacuum while absorbing up to $250 \mathrm{~kW}$ of synchrotron radiation power along both sides of its $25-\mathrm{m}$ length. It is being machined in the LLNL shop from solid copper plates. To preserve the material properties of the chamber, its top and bottom sections will be electron-beam welded at SLAC. Pumping slots on both sides of the dump provide high conductance to a novel NEG pumping configuration, shown in Fig. 5. In this pump, the NEG strips are cut into square "washers" and strung together on a support tube. A calrod heater is inserted into the central tube to activate the NEG material. In addition to its high linear pumping speed and high capacity, a big advantage of the design is that the heater element is external to the vacuum system and thus is easily replaceable if it fails.

In the interaction region, the vacuum configuration of the LER is quite complicated. Here the beam must be bent both vertically and horizontally, so the photon fans strike different surfaces in various sections of the chamber. To handle the power, we borrow here from the vacuum technology developed at SLAC for the HER [5]. We use copper extrusions, both the wide dipole chambers that house distributed ion pumps in the HER and the narrower quadrupole chambers without a DIP chamber. To provide adequate pumping speed, we again choose NEG pumps based on the design used in the wiggler (see Fig. 5). This choice is key to being able to achieve the low pressure (below 1 nTorr) required in the upstream portion of the IR.

It is worth commenting here that the use of NEG material in the LER does raise the 


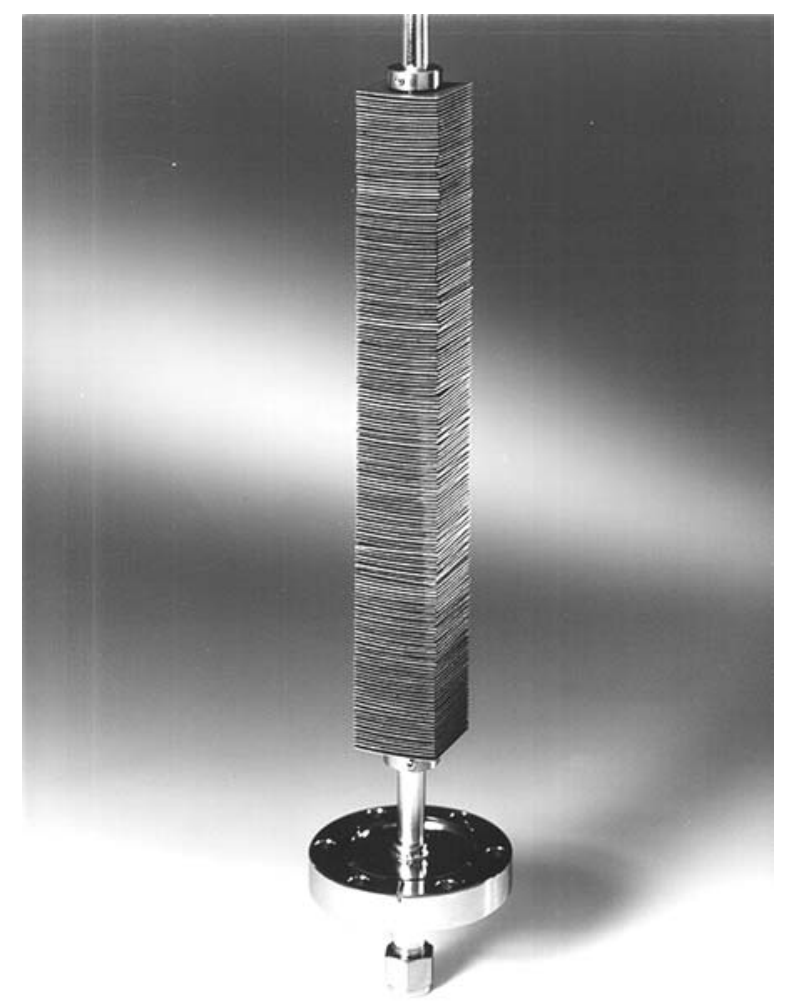

FIGURE 5. NEG pump prototype for wiggler and IR.

question of dust in the vacuum system, a problem that has been noted in some rings using NEG material. In an electron ring, the main worry is positively charged dust particles. This is not an issue for a positron ring such as the LER, but we still must be concerned with the possibility of magnetic dust particles from the NEG. We are working with the NEG manufacturer to avoid this possibility. A magnetic sieving of the raw NEG material is expected to suffice. Nonetheless, we are careful not to place the NEG material above the chamber where particulate matter might fall into the beam duct.

\section{RF System}

The RF system for PEP-II has been described in detail previously [6]. Briefly, we employ 476-MHz copper cavities with cavity HOMs damped via external broadband loads. The cavities are powered by commercially available 1.2-MW klystrons that feature high bandwidth and low group delay. (These features are needed because the klystron serves as part of the multibunch feedback system, damping the low coupled-bunch modes.) To minimize phase excursions of the colliding beams as the klystron reacts to the "ionclearing" gap, the transient responses of the LER and HER systems are matched. Thus, although both systems react to the gap, the collision point remains longitudinally stable.

In the LER, each klystron powers two cavities. A total of three stations (6 cavities) is installed to provide 5.1 MV.

\section{Feedback System}

The high beam current in the LER gives rise to strong coupled-bunch instabilities having 
growth times faster than $1 \mathrm{~ms}$. To combat this growth, we employ bunch-by-bunch feedback systems. These systems (longitudinal, horizontal, and vertical) give broadband response for all unstable modes.

In the longitudinal system [7], we use phase-offset detection to determine the beam motion. A farm of digital signal processors (DSPs) is used to define the filter algorithm that generates the required kick signal. The kick appropriate to damp the detected motion of each bunch is provided by broadband solid-state power amplifiers driving a drift tube kicker structure [8]. This approach can handle up to a $250-\mathrm{MHz}$ bandwidth and is sufficiently flexible that it can be used for many machines (PEP-II, ALS, DAФNE, or the BTCF).

A similar approach is used for the two transverse systems. However, here the digital portion of the system is restricted to providing the one-turn $(7.3 \mu \mathrm{s})$ delay. Two pickups are used in each plane to permit quadrature processing. This provides flexibility by making the system independent of the working point of the ring. To maximize the dynamic range of the kickers, we employ an orbit-offset rejection loop that ignores the static offset of the beam at the pickup locations. The vertical system also permits the kick phase to be inverted for any selected bunch, thus providing a single-bunch kickout feature. Simulations including machine nonlinearities showed that this kickout technique would be effective only in the vertical plane, as the horizontal motion decoheres too rapidly at large amplitudes to drive the beam out.

\section{Diagnostics}

To achieve "factory-like" performance, we have paid considerable attention to the diagnostics of the rings. The beam position monitor (BPM) system uses button electrodes to detect beam position at each quadrupole. After studying simulations, we decided to give most of the BPMs single-plane readout capability ( $x$-only at the QFs, $y$-only at the QDs). In selected locations, such as the IR, dual-plane readouts are provided. Each four-button BPM will be fiducialized and calibrated electrically to relate its electrical and mechanical axes. The measured offsets will be stored in the control system database. The BPM system will be capable of capturing single-turn orbit data, which will be a great aid in commissioning the ring.

To monitor injection, we are providing a bunch current monitor to look at the intensity of individual bunches. The system will use a dedicated BPM unit and will digitize each bunch with a $500 \mathrm{MHz}$ ADC. We plan to digitize every RF bucket, populated or not, to look for unwanted bunches that might accidentally get populated during the injection process. Our plan is to couple this system with the injection system to permit tailoring the bunch charges to optimize the beam-beam tune shift. The system will also serve to identify individual bunches with poor lifetime. This feature would be used, for example, to diagnose beam-beam problems by correlating beam losses with position along the bunch train.

Another tool we expect to be useful during the commissioning process is the beam loss monitor (BLM) system. Our design is based on small phototubes that can be read out by the control system computer. This approach gives us a linear response and a very broad dynamic range for looking at beam losses anywhere in the ring. The detectors are easily portable and can be located in any suspected problem area. They will permit us to identify possible problems with injection, bad vacuum, dust trapping, pressure bump instabilities, obstructions in the beam pipe, etc. Some detectors will be designated to monitor particular areas of the ring that might be damaged by the beam, such as the injection septum, collimators, and the detector. Depending on how reliably they work, some BLMs may be connected to the beam abort system as part of the machine protection system circuitry. 


\section{PROJECT STATUS}

As mentioned earlier, the PEP-II project is being built and commissioned in a phased manner. The injection system is well along, with most components fabricated and many of these installed and commissioned already. The HER is also well along, with most of its components in full production and being installed. The LER has begun production of most of its long-lead components and the IR is in the final design stage on its components. The IR design must be done in close collaboration with the $B A B A R$ detector design group, as the two systems must share a very confined spatial region within and near the detector.

\section{Injection System}

The injection system is based on extracting the electron and positron beams from the linac at their nominal energies and transporting them in separate bypass transport lines to the existing transport tunnels where they join the rings. At present, the two bypass lines have been installed side-by-side along the ceiling of the linac enclosure. The electron extraction area and bypass line have been commissioned and are operational. Components for the positron extraction are being completed and will be installed during this summer's shutdown of the SLC program.

\section{High-Energy Ring}

The HER makes use of the refurbished magnets from PEP. Refurbishment activities are essentially done and most of the magnets have been remeasured. All of the dipoles have been reinstalled in the HER arcs and the quadrupole rafts (containing a quadrupole, sextupole, corrector, and BPM unit) are being integrated and installed as they are available. The installation of an arc quadrupole raft is shown in Fig. 6.

The HER arc vacuum system is based upon copper extrusions [5]. Two types are employed, a wider dipole chamber, about $6 \mathrm{~m}$ long, that houses a DIP separated from the circulating beam by a slotted screen, and a narrower quadrupole chamber, about $2 \mathrm{~m}$ long, having the same cross section for the beam duct as the dipole chamber but without the

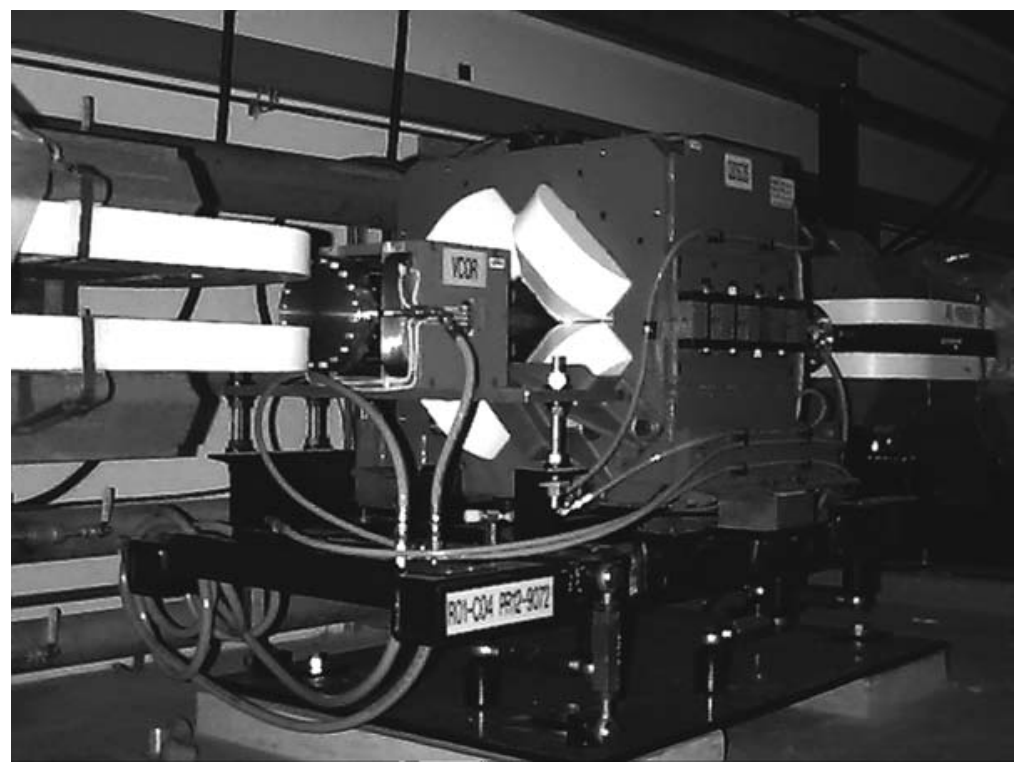

FIGURE 6. HER arc raft installed in the PEP tunnel. A vertical corrector magnet is included on the raft. 
DIP chamber and screen. To preserve the materials properties of the copper extrusions, the cooling tubes, DIP screen, BPM buttons, and flanges are attached by means of electronbeam welding. This necessitated acquiring a large electron-beam welder with a UHV compatible vacuum system. This device was installed at SLAC and is being used to manufacture the chambers in-house. (In addition to production of the HER arc chambers, the welder will be employed for the special chambers in the LER portion of the IR and for the LER wiggler chamber, which is machined from solid copper plate.) The HER vacuum work is now in full swing, with the LER work scheduled to begin immediately after it is completed. Straight section vacuum chamber production work is also under way [9]. The stainless steel chambers and pump tees are being manufactured in the SLAC shops and the vacuum processing and integration are done at LLNL. The same technology will be employed for the LER, whose components will be fabricated after completion of the HER work.

\section{Low-Energy Ring}

LER magnet production is now well along. The dipole and quadrupole production is being carried out by Berkeley Lab in collaboration with the Institute of High Energy Physics (IHEP) in Beijing. At present, about 80 of 320 required quadrupoles have been completed. Figure 7 shows an arc quadrupole undergoing magnetic measurements at IHEP. The field quality of the quadrupoles, as measured both at IHEP and at Berkeley Lab, has been better than the specified values. The prototype arc dipole has been completed (Fig. 8) and measured at both laboratories. It has met its design specifications and has been released for production.

LER sextupoles are based on refurbished cores from PEP sextupoles. New, smaller, coils are required to accommodate the magnet chamber extrusion. The new sextupole coils are being fabricated in industry and delivery is well along. The sextupoles are being rebuilt with the new coils as they arrive. Horizontal and vertical corrector dipoles are also fabricated in industry. About two-thirds of the required magnets have now been delivered to Berkeley Lab.

We have completed prototypes of the rafts and support structures for both the arcs and straight sections. The prototype arc raft, installed in the PEP tunnel, is shown in Fig. 2. The arc raft support system is shared with that for the HER dipoles, with an additional vertical support on the aisle side. Production of the full complement of LER rafts and supports is just getting under way.

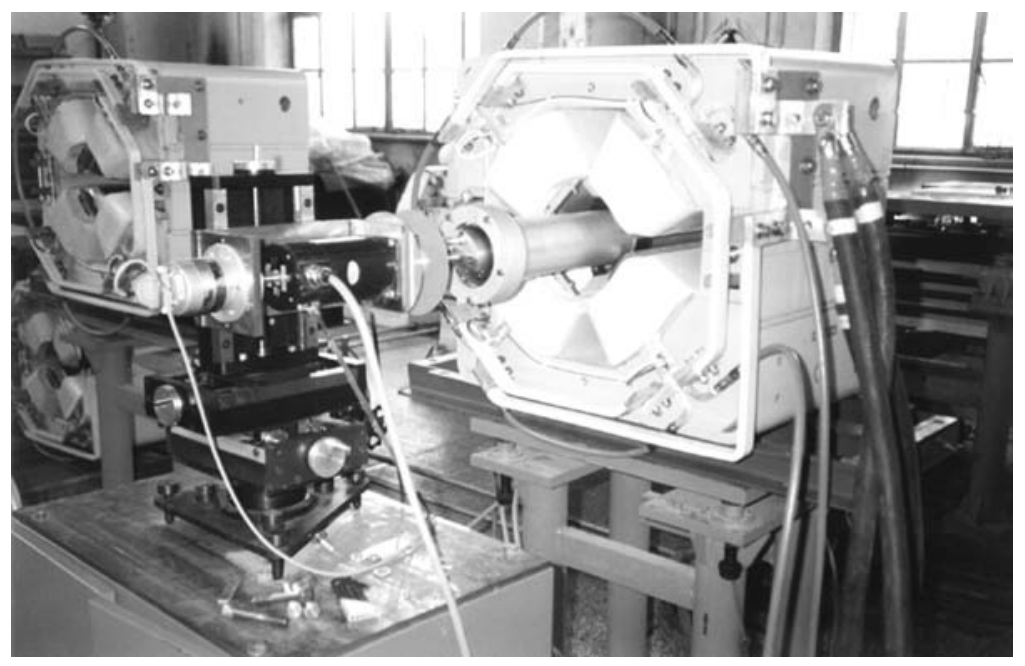

FIGURE 7. LER arc quadrupole undergoing magnetic measurement at IHEP with a rotating coil. 


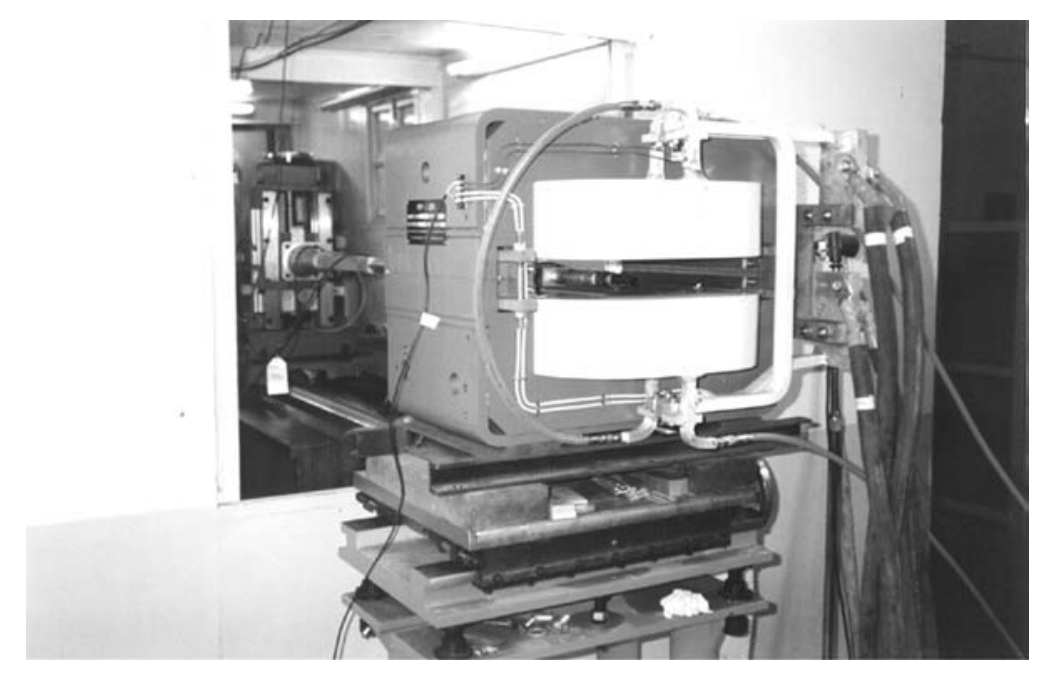

FIGURE 8. LER prototype arc dipole magnet undergoing magnetic measurements at IHEP.

In the vacuum area, the main emphasis presently is on the arc chambers. All required magnet chamber extrusions have been completed and delivered to Berkeley Lab. Figure 9 shows a prototype magnet chamber. The contract for machining the weld preps has been let and initial tests are beginning. Aluminum flange production is also under way. The welding of the production magnet chamber flanges will be carried out at Argonne National Laboratory, using equipment and techniques originally developed for the vacuum system of the Advanced Photon Source. All extrusions for the much larger pumping chamber have also been completed and delivered. The photon stop, a key component of the pumping chamber, has been successfully prototyped. The full order for the Glidcop material has been placed. Figure 10 shows the hot wall for the prototype photon stop prior to brazing. The serpentine cooling channels are machined into the plate, along with grooves for the wire braze material and an air-guard channel to serve as a buffer between the brazed water channels and the ring vacuum. The bottom of the hot wall (the surface seen by the photon beam) is also grooved to spread out the power from the photons. This serves to lessen the peak stress and temperature of the photon stop.

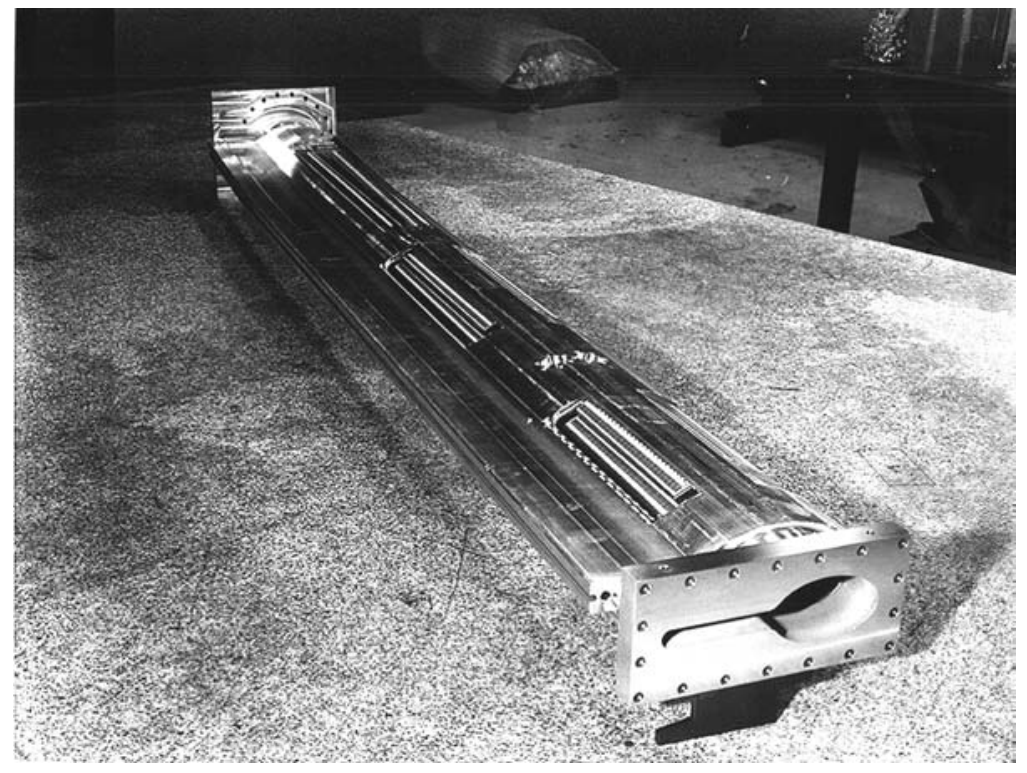

FIGURE 9. Prototype LER arc magnet chamber. The cut-outs accommodate magnet poles. 


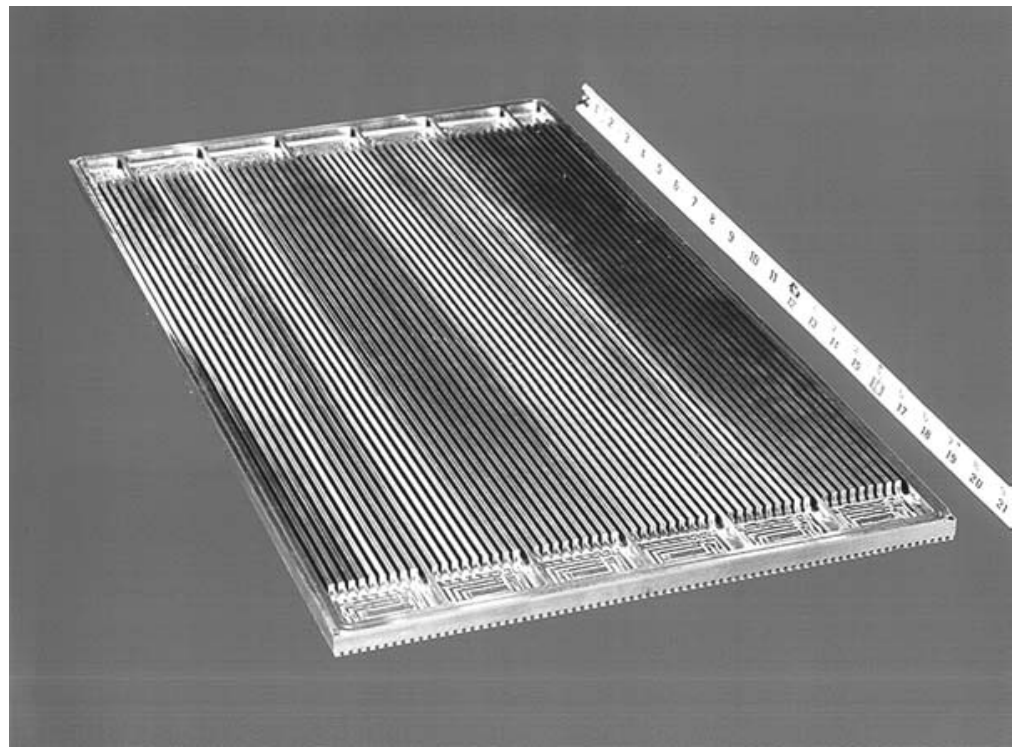

FIGURE 10. Glidcop hot wall of LER arc photon stop. Grooves on top serve as water channels. Grooves on bottom where photons strike serve to spread out the heat load and reduce peak stress. A backing plate is brazed to the upper surface to form the vacuum seal.

\section{Interaction Region}

This area [10] will be the last portion of the rings to be installed. Though HER commissioning will begin next year, the B1 and Q1 magnets will not be needed to characterize this ring and they will not be installed in this period. The absence of Q1 may slightly limit the ability to reach the very lowest beta function for which the HER is designed, but will not prevent our reaching its nominal operating parameters.

At the present time we are concentrating on the Q1 magnet, as this is the most challenging of the permanent-magnet devices. Our design calls for an offset magnet, and will be implemented by having a concentric dipole permanent magnet ring outside the quadrupole ring. The magnetic axis of the quadrupole is displaced horizontally by $2 \mathrm{~cm}$. Magnetic material $\left(\mathrm{Sm}_{2} \mathrm{Co}_{17}\right)$ for a prototype "slice" of Q1 has been ordered and will be used to assemble and magnetically measure a portion of the magnet. To adjust the strength of Q1 we will employ a water-cooled quadrupole winding capable of changing the integrated quadrupole strength by $\pm 3 \%$.

After a study of alternative designs, we have adopted a conventional iron-copper septum quadrupole for the Q2 magnet. This magnet (see Fig. 3) is the second member of the final focus doublet for the LER; the HER beam passes through the field-free region beyond its septum. Magnetic shielding between the LER and HER beams is a serious issue here, as the two beams are very close together. This will be included in the magnetic design of Q2. Magnetic shielding is also an issue with regard to the detector solenoid field. Field leaking out of the detector end caps will induce a skew octupole field in the Q2 magnets, especially that on the downstream HER side. To a lesser extent, the Q4 magnets suffer from the same problem. Mirror plates, possibly with small bucking coils, will be used to cope with this.

Detailed design work on the supports and vacuum chambers is also under way. This work must take account of the tight spatial constraints in the region and requires careful integration with the detector designers. One significant consideration we must include in the design is that of earthquake survivability. The $B A B A R$ detector will be mounted on isolators to minimize its motion in an earthquake. Because the machine components are separately supported, there can be considerable relative motion in a large earthquake. Our design must accommodate this motion without major damage to either machine or detector 
components.

\section{RF System}

The RF system of PEP-II is a major component of the project. The 1.2-MW klystrons must have special properties to serve as part of the multibunch feedback system. They have been designed to have a large bandwidth $(3 \mathrm{MHz})$ and a low group delay $(<150 \mathrm{~ns})$. The required tubes are being built by Philips in Hamburg, Germany. The first tube has been tested and met its design specifications. It has been delivered to SLAC for final testing. Full production of the required complement of eight tubes is now under way. High-power circulators have also been fabricated and delivered to SLAC.

After exploring other alternatives, it was decided to fabricate the RF cavities within the collaboration. LLNL is fabricating the cavity bodies and SLAC is providing the ancillary components (tuners, couplers, HOM loads, RF windows). The first production cavity is in the process of being tuned to the correct frequency. An initial complement of eight cavities, to be powered by two klystrons, will be installed later this year in preparation for HER commissioning next spring.

Tests of the RF cavity have been very encouraging. The test cavity has operated with $120 \mathrm{~kW}$ wall power dissipation, the full operating requirement. The RF window has demonstrated its design value power throughput of $500 \mathrm{~kW}$. We have used a $714-\mathrm{MHz}$ Damping Ring klystron to test the HOM loads, with satisfactory results. Tests of the prototype tuner have indicated problems with arcing in the spring fingers and we are examining alternative approaches to improve their design.

\section{Feedback Systems}

As has been reported before, the bunch-by-bunch feedback systems have been demonstrated under realistic conditions at the ALS. In these tests several important issues came to light. With the higher peak current that arises when the longitudinal feedback system is operating, heating problems were discovered in several components. One of the "flex-bands" that serve as bellows RF shields in the ALS heated up enough to melt one of the strips. Heating was also detected at the ends of the ceramic kicker magnet chambers. Both of these problems surfaced at lower peak currents than will occur in PEP-II and serve to emphasize the need for great care in the design of all machine components.

Components for the PEP-II systems are well along. The longitudinal feedback system is being designed and built at SLAC; the transverse systems are a Berkeley Lab responsibility. The broadband high-power amplifiers required for the longitudinal feedback system are ordered, and those for the transverse system are already delivered and tested.

Prototype kickers for both systems have been built. We have been measuring the impedance of these devices to look for trapped modes that have the potential for absorbing substantial amounts of HOM power from the beam. The longitudinal kicker is estimated to absorb only moderate power for the HER beam parameters, and it appears that it can be cooled radiatively by blackening its electrode surfaces. However, the power due to the higher beam current of the LER cannot be safely dissipated this way. A modified design of the kicker incorporating $\mathrm{BeO}$ stand-offs to conduct the heat away is being completed now. This approach will not significantly affect the electrical properties of the kicker.

\section{SUMMARY}

As should be obvious from the information provided here, we have made considerable 
progress in the design and construction of PEP-II in the past year. The injection system is mostly installed and partially commissioned, the HER is partially installed and in full production on all major components, the LER is in production on its long-lead items (magnets, vacuum components) and in the final design stage for most other components, and the IR is in the prototype phase.

The PEP-II project has an experienced, high-quality engineering, design, and accelerator physics team in place. This team is provided by all three collaborating labs, aided by our collaborators at IHEP and at the Budker Institute of Nuclear Physics in Novosibirsk. We are looking forward to continuing the phased commissioning process with the HER in April 1997, followed by the LER and IR one year later.

\section{ACKNOWLEDGMENTS}

The construction of a complex project such as PEP-II requires special dedication and skill from its staff. The success we have had to date is a testimony to their effort. I would especially like to acknowledge the outstanding System Engineers for the LER, Ron Yourd and Hank Hsieh for their help, advice, and guidance in building this complex and challenging system.

\section{References}

[1] PEP-II: An Asymmetric B Factory, Conceptual Design Report, ed. M. Zisman, SLAC-418, June 1993.

[2] M. Sullivan et al., "The Beam Stay Clear Definition of the PEP-II B Factory," to be published in the proceedings of the European Particle Accelerator Conference, EPAC'96, Sitges, Spain, June 1996.

[3] M. Furman and G. Lambertson, "The Electron-Cloud Instability in PEP-II," to be published in the proceedings of the European Particle Accelerator Conference, EPAC'96, Sitges, Spain, June 1996.

[4] J. Heim et al., "Wiggler Insertion of the PEP-II B Factory LER," in Proc. of 1995 Particle Accelerator Conf., Dallas, May, 1995, p. 527.

[5] C. Perkins, et al., "Vacuum System Design for the PEP-II B Factory High-Energy Ring," in Proc. of the European Particle Accelerator Conference, EPAC'94, London, England, June 27-July 1, 1994, p. 2482.

[6] H. Schwarz and R. Rimmer, "RF System Design for the PEP-II B Factory," in Proc. of 1995 Particle Accelerator Conf., Dallas, May, 1995, p. 1882.

[7] G. Oxoby et al., "Bunch-by-Bunch Longitudinal Feedback System for PEP-II," in Proc. of the European Particle Accelerator Conference, EPAC'94, London, England, June 27-July 1, 1994, p. 1616.; J.D. Fox et al., "Operation and Performance of a Longitudinal Damping System Using Parallel Digital Signal Processing," ibid., p. 1619.

[8] J.N. Corlett et al., "Longitudinal and Transverse Feedback Kickers for the ALS," in Proc. of the European Particle Accelerator Conference, EPAC’94, London, England, June 27-July 1, 1994, p. 1625.

[9] U. Wienands et al., "The Vacuum System for the PEP-II High Energy Ring Straight Sections," in Proc. of 1995 Particle Accelerator Conf., Dallas, May, 1995, p. 2129.

[10] M. Sullivan et al., "Interaction Region Design at the PEP-II B Factory," to be published in the proceedings of the European Particle Accelerator Conference, EPAC'96, Sitges, Spain, June 1996. 